\title{
Conservación de las propiedades nutraceúticas del Aloe vera (Aloe barbadensis Miller), mediante técnicas de secado
}

\section{Conservation of aloe vera nutraceutical properties (Aloe barbadensis Miller) by drying techniques}

\author{
Kerly Lorena Artunduaga Antury \\ https://orcid.org/0000-0002-4400-6266 \\ Estudiante, Ingeniería Agrícola, Universidad Surcolombiana. Email: u20132123473@usco.edu.co \\ Diego Andrés Vargas Rojas \\ https://orcid.org/ 0000-0003-3458-3729 \\ Estudiante, Ingeniería Agrícola, Universidad Surcolombiana. Email: u20132123607@usco.edu.co \\ Oscar Mauricio Barrera Bermeo \\ https://orcid.org/ 0000-0002-1837-046X \\ Magíster en Ciencia e ingeniería de los alimentos. Docente. Universidad Surcolombiana \\ Email: oscar.barrrera@usco.edu.co \\ Fecha de revisión:01/02/2021
}

Fecha de recibido:13/11/2020

DOI: $10.25054 / 22161325.2818$

\section{Resumen}

Se realizó el análisis de las aplicaciones y técnicas de secado para la obtención de polvo de aloe vera, usado en la elaboración de productos alimentarios, farmacéuticos y cosméticos. Para lo anterior, se tuvo en cuenta las técnicas existentes y aquellas que permiten la mayor conservación de sus propiedades nutracéuticas y funcionales para la elaboración de estos productos, garantizando inocuidad y seguridad para el consumidor. Se realizó una descripción respecto al funcionamiento y algunas pautas de los equipos, seleccionando el método de deshidratación más adecuado para producir un producto de alta calidad. Para el desarrollo de éste, se consultaron las principales bases de datos como Science Direct, EBSCO, Springer Journals, Scopus, ProQuest Central, Redalyc, Wiley Online Library entre otras. Se determinó, que las técnicas que permite la mayor conservación de las propiedades del aloe vera fueron: secado por aspersión, liofilización y ventana refractante, debido a las bajas temperaturas usadas, la cual evita la degradación de sus componentes (dada a la alta sensibilidad del gel a temperaturas superiores a $50^{\circ} \mathrm{C}$ ).

Palabras clave: Funcional; trasformación; estabilización; vida útil.

\begin{abstract}
An analysis was made of the applications and drying techniques for obtaining aloe vera powder, used in the production of food products, pharmaceuticals, and cosmetics. For the above, the existing techniques and those that allow the greater conservation of their nutraceutical and functional properties were taken into account, for the elaboration of these products, guaranteeing safety and safety for the consumer. A description was made regarding the operation and some guidelines of the equipment, selecting the most appropriate dehydration method to produce a high-quality product. For the development of this, the main databases were consulted such as Science Direct, EBSCO, Springer Journals, Scopus, ProQuest Central, Redalyc, Wiley Online Library among others. It was determined that the techniques that allow the best conservation of the properties of aloe vera were: spray drying, freeze-drying and refracting window, due to the low temperatures used, which prevents the degradation of its components (given the high sensitivity of the gel at temperatures above $\left.50^{\circ} \mathrm{Celsius}\right)$.
\end{abstract}

Keywords: Functional; transformation; stabilization; shelf life. 


\section{Introducción}

El gel de aloe vera o sábila (conocido comúnmente), es un producto reconocido a nivel mundial, debido a alto contenido de compuestos bioactivos como: flavonoides, terpenoides, lectinas, ácidos grasos antraquinonas, minerales y vitaminas que se relacionan con una respuesta biológica antiviral, cicatrizante (heridas), antitumoral, fortalecimiento del sistema inmune, para el cuidado de piel, efectos hepatoprotectores y antidiabéticos, (Canche-Escamilla et al., 2019; Maan et al., 2018; Nicolau-Lapeña et al., 2021), por lo cual, tiene aplicaciones en la industria cosmética, alimentaria y farmacéutica (Maan et al., 2018; Santacruz et al., 2015). El aloe vera (Aloe barbadensis Miller) es una planta suculenta perenne perteneciente a la familia Aloaceae, que se ha utilizado por más de 2000 años por sus propiedades curativas y terapéuticas (Reyes et al., 2012), el 98.5-99.5\% de la planta corresponde a agua (Sriariyakul et al., 2016) y el restante, se compone principalmente de polisacáridos (más de $60 \mathrm{~g}$ de polisacáridos / $100 \mathrm{~g}$ de sólidos de gel) (R. Minjares et al., 2016); además, contiene aproximadamente 75 nutrientes, 200 compuestos activos que incluyen aminoácidos, azúcares, enzimas, vitaminas, minerales, saponinas, antraquinonas, lignina y ácido salicílico (Maan et al., 2018).

Alrededor de 42 mil hectáreas son cultivadas en América (19 mil) y en el mundo (23mil), cada año. Los principales exportadores de Aloe vera son: Tailandia (35\%), México (30\%) y República Dominicana (18\%) (Bahmani et al., 2016). La producción en Colombia, de acuerdo a la cadena productiva sábila (2017), cuenta con un área sembrada de 1815 ha, en los departamentos: Valle del Cauca, Risaralda y Huila, con una producción del $22 \%, 17 \%$ y $5 \%$, respectivamente.

Actualmente, el consumo de alimentos con propiedades benéficas para la salud, ha ido en aumento (Lozano et al., 2011); por ello, las distintas industrias están interesadas en la generación de nuevos productos que tengan un efecto positivo sobre la salud del consumidor. Debido a los componentes bioactivos presentes en el Aloe vera.es usada en la elaboración de bebidas con aloe, leche, helados, suplementos alimenticios, preparaciones de gel, pomadas, cremas, jabones, champús, limpiadores faciales, lociones)
(Sánchez et al., 2017), (Mudgil et al., 2016). Por lo tanto, optimizar la cadena productiva en su recolección, manejo, transporte, molienda, deshidratación, extracción, y estabilización del gel, es fundamental para obtener productos que conserven las propiedades fisiológicas y farmacéuticas de esta planta (Sánchez et al., 2017).

Así mismo, factores ambientales favorecen la rápida oxidación, descomposición y pérdida de sus actividades biológicas, cuando el gel es expuesto a ellos, contribuyendo a la degradación de la matriz de gel por reacciones enzimáticas naturales, como al crecimiento de bacterias, debido a la presencia de oxígeno (Ramachandra \& Srinivasa, 2008). Sin embargo, investigadores han desarrollado técnicas que ayudan en la esterilización y estabilización durante su procesamiento, usando tratamientos térmicos.

Por otro lado, el gel Aloe vera es altamente perecedero, por lo que el uso de las tecnologías apropiadas es fundamental con el fin de aumentar la estabilidad de almacenamiento, conservando sus propiedades funcionales (R. Minjares et al., 2016) . La deshidratación (secado), es uno de los métodos comúnmente aplicado para la conservación del producto, en el sector agrícola y farmacéutico. Mediante el secado se elimina líquido para obtener un producto seco con los criterios de calidad correspondientes (Rosero, 2018). Sin embargo, según Vega et al., (2007) y Miranda et al., (2009) citado por Domíguez et al., (2012), ha evidenciado en sus investigaciones el efecto de la temperatura en los parámetros cinéticos del secado convectivo del gel, en donde rangos de temperatura de 50 a $90{ }^{\circ} \mathrm{C}$ reducen el tiempo de secado de 800 a 200 minutos para llegar a la humedad de equilibrio específica y a la vez disminuye la capacidad antioxidante del gel al aumentar la temperatura.

El siguiente documento, tiene como objetivo analizar e identificar cuál es el método de secado que permite un mejor aprovechamiento de la materia prima, conservando sus propiedades funcionales y organolépticas al final del proceso, ya que éste es uno de los ingredientes base más utilizados en las industrias (alimenticia, farmacéutica y cosmética) por ser versátil en cualquier formulación; además, resaltar sus aplicaciones, composición química, técnicas de deshidratación, estabilización, entre otras. 


\section{Materiales y métodos}

\subsection{El Aloe Vera (Aloe barbadensis Miller)}

Existen alrededor de 350 especies de aloe, sin embargo, las más populares son el aloe vera (Aloe barbadensis Mill.) y el árbol de aloe (Aloe arborescens Mill.). Los geles de las especies de éste último y Aloe ferox, mostraron actividad farmacológica en la cicatrización de heridas (BialikWąs et al., 2021; Guillén et al., 2013; Solek et al., 2018)

El género aloe, es una hierba suculenta de 80 - 100 $\mathrm{cm}$ de altura que madura en 4 - 6 años y sobrevive durante casi 50 años en condiciones favorables (Sahu et al., 2013). La planta puede sobrevivir a una temperatura de $40{ }^{\circ} \mathrm{C}$ y por debajo de la temperatura de congelación, dependiendo de la salud de la raíz (Baruah et al., 2016).

El Aloe vera es una planta perenne con hojas verdes turgentes unidas en el tallo en forma de roseta. Las hojas están formadas por una epidermis gruesa (piel) cubierta con cutículas que rodean la mesofila, que se pueden diferenciar en células de clorénquima y células de paredes más delgadas que forman el parénquima (R. Minjares \& Femenia, 2019) y crece en las regiones como: África, Asia, Europa y América (Bajpai, 2018)

\subsection{Composición química}

El Aloe vera está compuesto por cerca de 20 sustancias favorables para la salud (Domíguez et al., 2012). En materia seca, el gel de aloe se compone de polisacáridos $(55 \%)$, azúcares $(17 \%)$, minerales (calcio, cromo, cobre, hierro, magnesio, manganeso, potasio, fósforo, sodio, zinc) (16\%), proteínas $(7 \%)$, lípidos $(4 \%)$ y compuestos fenólicos $(1 \%)$, vitaminas (A,C, E, $\beta$-caroteno, B1, B2, B3, B6, B12, ácido fólico) (Ahlawat \& Khatkar, 2011; Nicolau-Lapeña et al., 2021).
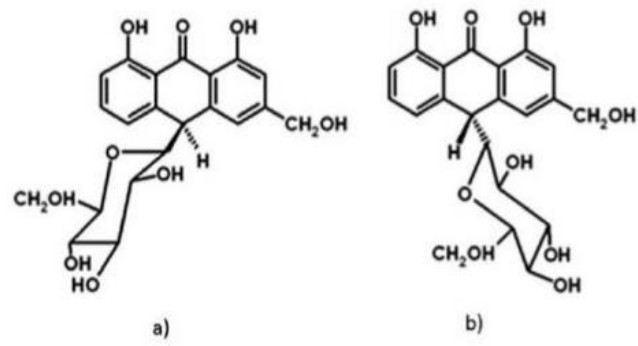

Figura 1. Estructura a) Barbaloíona, b) Isobarbaloina (Lacerda, 2016)

Las cromonas y las antraquinonas (barbaloína e isobarbaloina), son los principales compuestos fenólicos del Aloe vera (Figura 1) (Lacerda, 2016). La aloína es el principal componente del acíbar, que la planta secreta como defensa para alejar a posibles depredadores por su olor y sabor desagradable (Domíguez et al., 2012).

\section{Resultados y discusiones}

\subsection{Usos y aplicaciones del aloe}

Alimentos nutracéuticos, definido como una sustancia alimenticia o parte de ella, que proporciona beneficios para la salud, en la prevención de enfermedades. La mayoría de éstos, no son productos farmacéuticos y, por lo tanto, se tratan como alimentos (Khorasani et al., 2018). Se ha descubierto que los nutracéuticos desempeñan un papel clave en diferentes funciones fisiológicas en el cuerpo y ayudan a combatir varios trastornos como enfermedades cardiovasculares, cáncer, diabetes, hipertensión, entre otros. La funcionalidad de los alimentos es valorada cuando éstos compuestos están presentes (Nazir et al., 2019).

Dado los diferentes beneficios del aloe vera, hacen que sea un producto de interés para la industria farmacéutica y alimentaria. En la figura 2, se representa la distribución del Aloe Vera en la industria (Patiño, 2016). 


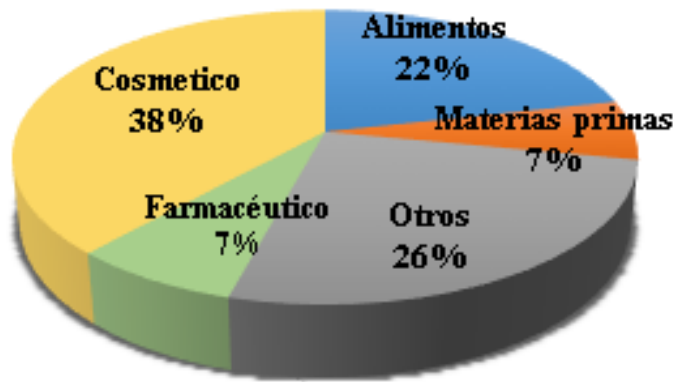

Figura 2. Usos del Aloe vera según la industria (Patiño, 2016)

\subsection{Industria alimentaria}

En la actualidad, el Aloe vera barbadensis Mill y Aloe vera arborescens han sido utilizados en la elaboración de recubrimientos de frutas (uvas, durazno, ciruelas, cerezas), debido a que reducen la pérdida de peso y la producción de etileno, manteniendo la calidad e inhibiendo la proliferación de microorganismos (Penicillium, Botrytis , Monilia) (Ortega et al., 2017). Un estudio reciente en kiwis cortados en el que se usó Aloe vera, fue eficaz para reducir la despolimerización de pectina y la proliferación microbiana; además, mejoró la calidad sensorial (Benítez et al., 2015).

Éste tipo de recubrimiento protege y conserva, contra la transferencia de vapor de agua, oxígeno y dióxido de carbono, retrasando la maduración, mejorando las propiedades organolépticas (sabor, aroma, textura y apariencia) (Khaliq et al., 2019) (Amanullah et al., 2017). Adicionalmente, es una alternativa biodegradable al uso de envases de plástico (Vieira et al., 2016).

Generalmente, el gel de aloe se utiliza en la preparación de bebidas saludables como la leche, helados, confitería (Mishra \& Sangma, 2017). La tabla 1, muestra las aplicaciones del Aloe vera en la industria alimentaria.
Tabla 1. Aplicaciones alimentarias de los productos de Aloe vera

\begin{tabular}{cl}
\hline Productos & \multicolumn{2}{l}{ Aplicaciones Alimentarias } \\
\hline \multirow{3}{*}{ Concentrado jaleas, } & $\begin{array}{l}\text { Mermelada, } \\
\text { concentrado para mezclar con } \\
\text { té, agua o jugo. }\end{array}$ \\
\hline \multirow{2}{*}{ Jugo } & $\begin{array}{l}\text { Bebida saludable, bebida } \\
\text { laxante, sorbete, bebida } \\
\text { deportiva, dietética con fibra } \\
\text { soluble, yogures, mezcla de } \\
\text { Aloe vera para whisky u otro } \\
\text { alcohol, pan blanco con Aloe } \\
\text { vera. }\end{array}$ \\
\hline & $\begin{array}{l}\text { Yogur, cuajada, 'lassi', helado } \\
\text { y Aloe vera 'laddu'. }\end{array}$ \\
\hline Filete de gel & $\begin{array}{l}\text { Caramelos, barra, bocadillo, } \\
\text { goma de mascar, gránulos de } \\
\text { té instantáneo, } \\
\text { vitaminas tipo caramelo, } \\
\text { batidos de frutas. }\end{array}$ \\
\hline
\end{tabular}

(Ahlawat \& Khatkar, 2011)

\subsection{Industria farmacéutica}

El Aloe vera se ha utilizado para la fabricación de productos de uso tópico como ungüentos y geles, así como en la producción de capsulas (tabla 2). El extracto de hoja (penca) incluye la capacidad de mejorar la biodisponibilidad de vitaminas coadministradas en personas, debido a sus efectos potenciadores de la absorción eficaz de medicamentos poco absorbibles, a través de la vía oral. Además, el producto seco en polvo obtenido del gel, ha sido utilizado con éxito para fabricar tabletas de tipo matriz directamente comprimible (Srujana et al., 2012). 
Tabla 2. Resumen de las aplicaciones farmacológicas del Aloe vera

\begin{tabular}{cc}
\hline Efecto & $\begin{array}{c}\text { Parte de la } \\
\text { planta usada }\end{array}$ \\
\hline Apósito para heridas & Hoja \\
\hline Tratamiento del vértigo & Hoja \\
\hline Curación de heridas & Aloe gel \\
\hline Efecto antidiabético & Aloe gel \\
\hline $\begin{array}{c}\text { Cáncer de mama, } \\
\text { cáncer gastrointestinal } \\
\text { y glioblastoma cerebral }\end{array}$ & $\begin{array}{c}\text { Aloe vera }+ \\
\text { miel }\end{array}$ \\
\hline $\begin{array}{c}\text { Efecto antitumoral y } \\
\text { antiulceroso }\end{array}$ & $\begin{array}{c}\text { Glicoproteínas } \\
\text { de Aloe vera }\end{array}$ \\
\hline
\end{tabular}

(Ahlawat \& Khatkar, 2011)

\subsection{Industria cosmética}

Tabla 3. Productos cosméticos comerciales ricos en polifenoles disponibles en el mercado

\begin{tabular}{ll}
\hline \multicolumn{1}{c}{ Productos comerciales } & \multicolumn{1}{c}{ Contenido principal de polifenoles/extractos } \\
\hline & $\begin{array}{l}\text { Jugo de hoja de Aloe barbadensis (Aloe vera) orgánico, } \\
\text { extracto de hoja de Camellia oleifera (té verde japonés) } \\
\text { orgánico, extracto orgánico de Punica granatum } \\
\text { (granada), extracto orgánico de Hamamelis virginiana, } \\
\text { extracto orgánico de Vaccinium macrocarpon } \\
\text { (arándano), extracto, aceite de semilla orgánica de } \\
\text { Simmondsia chinensis (jojoba), aceite de semilla } \\
\text { orgánica de Argania spinosa (argán), pulpa de Cocos } \\
\text { nucifera (coco), vainilla tahitensis (vainilla), extracto de } \\
\text { fruta, entre otros. }\end{array}$ \\
\hline Poly-GRO & \multicolumn{1}{c}{ Procianidina B2 de la manzana } \\
\hline Caudalie Viña activa & Semillas de uva y extracto de Picea abies \\
\hline Polifenoles Caudalie & Semillas de uva \\
\hline Chantecaille & Extracto de manzana verde \\
\hline Whole blends Garnier & (Rodrigues et al., 2018)
\end{tabular}

\subsection{Estabilización del Aloe vera}

Durante el procesamiento del gel de Aloe vera, se pueden alterar las propiedades de los polisacáridos al afectar su estructura original, generando algunos cambios en las propiedades farmacéuticas de éstos,
De acuerdo a Javed \& Atta (2014), el gel de Aloe vera es un ingrediente cosmético y dermatológico ideal, utilizado en más del $95 \%$ de estos productos. Tiene preparaciones antienvejecimiento únicas para la conservación de una piel vigorosa y de aspecto fresco; se ha utilizado como material de base para la producción de cremas, lociones, jabones, champús, limpiadores faciales, entre otros (tabla 3) (Nejatzadeh, 2013). Adicionalmente, se usa ampliamente en los tejidos faciales, donde se promueve como un humectante y / o anti-irritante (Ray, 2012). También, como tónico de la piel contra granos y como hidratante para mantener la piel nebulizada en climas secos y evita la descamación del cuero cabelludo. La acción cosmética del gel de Aloe vera es antiinflamatoria, tonificante, calmante $\mathrm{e}$ hidratante (Javed \& Atta, 2014).

(Rodrigues et al., 2018)

debidas a las operaciones realizadas como: triturar, moler, presionar la hoja (penca), separación del gel del epicarpio y moler el gel para producir un jugo de aloe, seguido de varios pasos de filtración y estabilización. Además, su alta actividad hídrica de $\mathrm{A}$. vera $\left(\mathrm{a}_{\mathrm{w}}>0.90\right)$ 
y composición de carbohidratos, su vida útil es de 3 4 días a temperatura ambiente, por lo que es necesario utilizar métodos de conservación para mantener la mayoría de los ingredientes activos y aumentar su vida útil (Cervantes et al., 2014).

La estabilización del gel de Aloe vera suele obtenerse concentrando el gel para reducir el agua o secándolo para obtener polvo sólido. También, puede lograrse añadiendo conservantes y otros aditivos, por ejemplo, benzoato de sodio y ácido cítrico. Los beneficios para la salud de Aloe vera se atribuyen a sus actividades biológicas. El gel, se puede concentrar en vacío sin pérdida de actividades biológicas(Rahman et al., 2017). Por tanto, es imperativo que se desarrollen técnicas de procesamiento simple pero eficiente para mejorar la calidad del producto, preservando y manteniendo la mayor parte de los compuestos bioactivos presentes de forma natural.

A partir de lo anterior, se ha realizado una descripción y comparación de diferentes técnicas (tratamientos térmicos), utilizadas con dicho propósito.

\subsection{Técnicas de secado de productos agropecuarios}

El secado (o deshidratación) es una de las operaciones unitarias importantes en la elaboración de alimentos, para su conservación, al reducir la cantidad de humedad en la matriz alimentaria a niveles que ralentizarán e inhiben la actividad microbiana, enzimática y la calidad del producto asociada al deterioro. Éste es un proceso que consume mucha energía, que puede alterar los atributos de calidad asociados a largos periodos de exposición y altas temperaturas. El proceso de secado de materiales alimenticios es extremadamente complejo, e involucra mecanismos transitorios acoplados de procesos de transferencia de calor, masa y momento, acompañados por transformaciones físicas, químicas y de cambio de fase, reduce el volumen y masa para su almacenamiento y transporte (Sabarez, 2016).

Es importante considerar el comportamiento de las propiedades funcionales de la matriz del alimento frente a la exposición de temperatura, ya que algunos de éstos son altamente afectados por los procesos de secado, influyendo significativamente en el alcance de su aplicación y valor comercial (Dehnad et al., 2016).

Las tecnologías de secado pertenecen a la primera, segunda, tercera o cuarta generación. Las correspondientes a la primera generación son: los secadores de tipo gabinete y lecho, como: el horno, la bandeja, el transportador de flujo rotativo y el túnel que utilizan aire caliente como medio de transferencia de calor. Los secadores por aspersión y tambor, de segunda. Y a la tercera, pertenecen: liofilización y la deshidratación osmótica. Finalmente, secado por microondas, infrarrojos, bomba de calor, radiofrecuencia y ventana de refractancia a la cuarta (RW TM) (Raghavi et al., 2018).

El uso potencial de los productos de Aloe vera, a menudo implica algún tipo de procesamiento, incluyendo, entre otros, el secado. De éste último se obtiene materia seca (polvo), que puede mezclarse con otros ingredientes para formar una gran variedad de productos dietéticos, cosméticos y farmacéuticos (Krokida et al., 2012).

\subsection{Secado por convección forzada}

Actualmente, el uso del secado por convección (secado por aire caliente), se mantiene como una como un método para la conservación de alimentos. En el cual, el calor ingresa a la matriz alimentaria por una corriente de aire caliente, donde es transferido a la superficie del producto por convección y luego dentro del mismo por difusión o convección, dependiendo de su estructura. El flujo de calor provoca un aumento de la temperatura del producto y en consecuencia la evaporación del agua (Castro et al., 2018). Los secadores por convección, se puede operar a bajas temperaturas, para proteger el material contra el sobrecalentamiento, la contracción, la decoloración y el endurecimiento de la carcasa. Un factor importante es el efecto de la exposición a largo plazo a altas temperaturas, que influye directamente en la calidad del producto induciendo degradaciones indeseables (Szadzińska et al., 2016) (Karam et al., 2016).

Según Vega et al., (2007) observó en el Aloe vera, que el tiempo de secado disminuyó cuando la temperatura aumentó hasta alcanzar humedad de equilibrio. Las temperaturas utilizadas fueron $(50,60$, 70,80 y $90^{\circ} \mathrm{C}$ ), analizando el contenido de humedad en función del tiempo. El tiempo requerido para lograr un contenido de humedad inferior a $2.0 \mathrm{~g}$ de agua $/ \mathrm{g}$ hbs a $50^{\circ} \mathrm{C}$ fue de 800 minutos, siendo el doble del tiempo requerido para alcanzar el mismo contenido de humedad a una temperatura de $70^{\circ} \mathrm{C}$ (400 minutos), y triple para una temperatura de $90^{\circ} \mathrm{C}(200 \mathrm{~min})$. 


\subsection{Secado por aspersión}

Es una tecnología ampliamente utilizada en los últimos años, conocido como Spray drying (SD), el cual permite que propiedades del producto como sabor, color y nutrientes, se conservan en altos porcentajes. El SD, es un método apropiado para productos sensibles al calor, como jugo de frutas, puré de verduras y productos químicos ricos en azúcares. Es una técnica de evaporación de agua que utiliza aire caliente para estabilizar soluciones y suspensiones para producir polvos ligeros, con un tamaño de partícula y contenido de humedad específicos, operación continua, tiempos de producción cortos, rentabilidad y flexibilidad (Medina et al., 2016) (Cervantes et al., 2014).

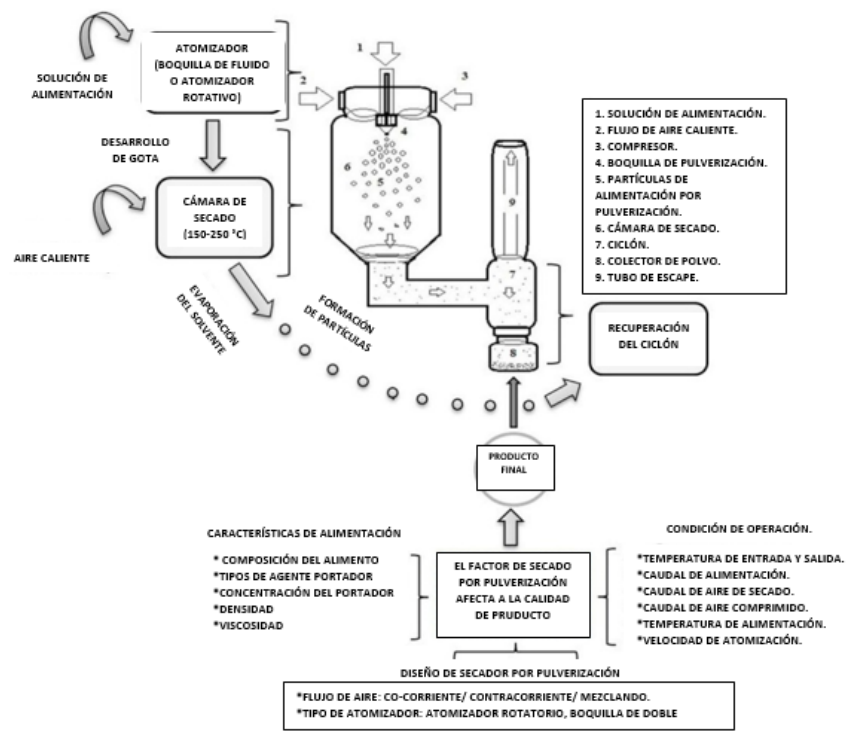

Figura 3.Diagrama esquemático del secado por aspersión y factores que afectan la calidad del producto (Shishir \& Chen, 2017)

El SD, consta de tres pasos: atomización de la muestra alimentaria, secado de las gotas de líquido y recuperación del polvo como se muestra en la figura 3. Durante la atomización, la alimentación líquida pasa a través de un atomizador a la cámara de secado y se distribuye en pequeñas partículas líquidas en un gran volumen. La atomización maximiza el área de volumen de superficie de la alimentación líquida para un secado efectivo y eficiente. Las propiedades del producto final dependen del diseño del atomizador y su rendimiento (Shishir \& Chen, 2017). Estudios realizados por Cervantes et al., (2014), mediante SD del Aloe vera en función de las propiedades reológicas, encontraron que tiene un alto potencial como espesante, estabilizador con aplicación industrial. Además, es un método que ayuda a prolongar la vida útil del aloe, preservando sus propiedades mecánicas para fluir. Otros autores, como Tonon et al (2008), citado por Shishir \& Chen (2017), encontraron que el tamaño de partícula del polvo de fruta açaí aumentó de $13.38 \mathrm{~mm}$ a $20.11 \mathrm{~mm}$ con el aumento de la temperatura de secado de entrada de $138^{\circ} \mathrm{C}$ a $202^{\circ} \mathrm{C}$.

\subsection{Secado por liofilización}

Conocida como Freeze-drying por sus siglas en inglés $\mathrm{FD}$, es una técnica de deshidratación que representa el proceso ideal para la obtención de productos secos de alto valor. Este método, realiza una congelación y se usa con frecuencia para lograr una estabilidad de almacenamiento a largo plazo de productos biofarmacéuticos sensibles. El proceso de FD consiste principalmente en dos pasos: el producto se congela a $\left(-20^{\circ} \mathrm{C}\right)$ y luego se aplica una cantidad controlada de calor al vacío (secado por congelación al vacío) o a presión atmosférica (secado por congelación atmosférica) para promover la sublimación (figura 4). Durante la FD, la eliminación de la humedad interna pasa por dos etapas: 1- produce la sublimación de los cristales de hielo y 2 - donde se produce la desorción del agua restante no congelada (Karam et al., 2016). Más del $40 \%$ de los productos biológicos comercializados son liofilizados. El proceso de fabricación del alimento, requiere mucho tiempo (días o semanas) y dinero (Horn et al., 2018).

Esta técnica es bien conocida por su capacidad para mantener la calidad del producto (color, forma, aroma y valor nutricional) superior a cualquier otro método de secado, debido a su baja temperatura de procesamiento entre $\left(2\right.$ a $\left.10{ }^{\circ} \mathrm{C}\right)$ y la ausencia virtual de oxígeno en el aire durante el procesamiento, lo que minimiza las reacciones de degradación. Otros factores prominentes, incluyen la rigidez estructural mostrada por la sustancia congelada en la superficie, así como la movilidad limitada del agua congelada evitando así colapsos y encogimientos de la matriz sólida. Sin embargo, es importante resaltar, que esta técnica es un método costoso. Es económicamente viable solo en el caso de productos de alto valor agregado y siempre que la calidad superior del producto justifique el mayor costo de producción (Berk, 2018). 


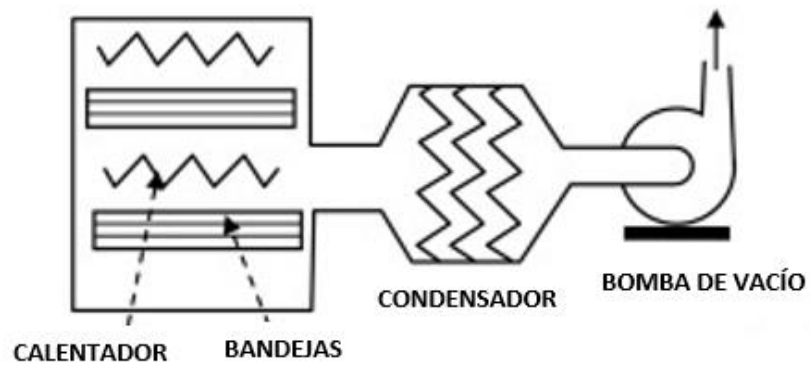

Figura 4. Partes de un liofilizador (Berk, 2018)

De acuerdo a Simal et al., (2000) citado por (Nindo et al., 2010) evaluaron los filetes secados por convección y liofilizados de las hojas de Aloe vera para determinar su capacidad de retención de agua, rehumedecimiento y capacidad de absorción de grasa, observaron que las temperaturas del aire $\left(40^{\circ} \mathrm{C}\right.$ a $80^{\circ} \mathrm{C}$ ) tenían un efecto mayor sobre esas propiedades que el tiempo de secado. Otros estudios sobre el secado del Aloe vera, han determinado el efecto de la temperatura y la humedad relativa sobre el tiempo de secado y los cambios en las propiedades fisicoquímicas y la actividad antioxidante.

\subsection{Secado por ventana refractiva}

Es una de esas técnicas que ha llamado la atención, debido a los numerosos beneficios. La técnica consiste en secar purés y líquidos colocados sobre una delgada película transparente infrarroja que esencialmente forma una "ventana" a través de la cual se produce el secado (figura 5). Es importante destacar que las temperaturas del producto se mantienen bajas (alrededor de $39^{\circ} \mathrm{C}$ ) y se produce un secado rápido, ya que están involucrados los tres modos de transferencia de calor (Raghavi et al., 2018).

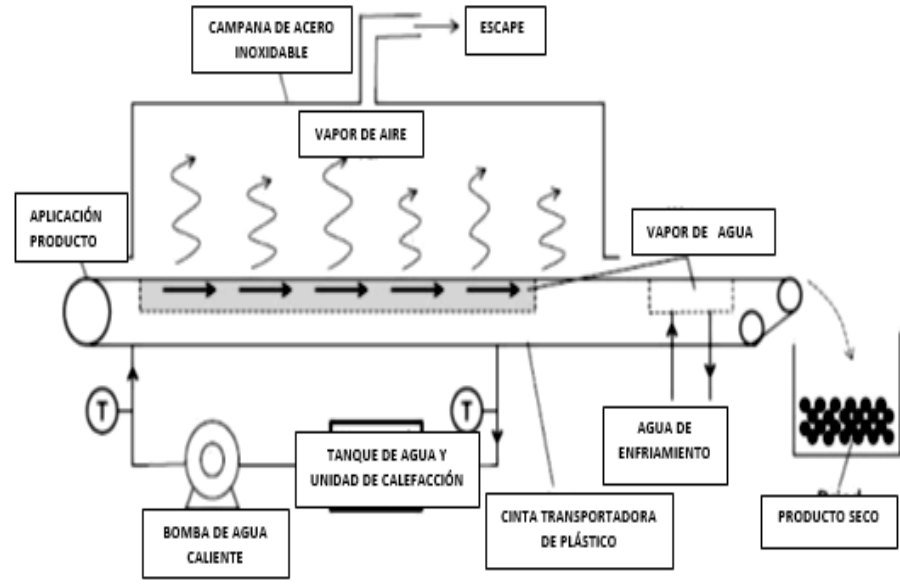

Figura 5. Diagrama esquemático de un sistema de secado RW TM (Raghavi et al., 2018)

Esta tecnología fue patentada por Magoon (1986) y desarrollada por MCD Technologies Inc. (Tacoma, Washington, EE. UU.). Conocida por la capacidad para obtener alimentos deshidratados de alta calidad en comparación con el proceso de secado convencional (secado por convección). Por lo que, éste método, no solo mejora el color, el sabor, sino también la retención de vitaminas y antioxidantes (Franco et al., 2019).

Las principales ventajas relacionadas con esta nueva técnica son: evita la contaminación cruzada ya que el medio de transferencia de calor no entra en contacto directo con el producto en sí, además se caracteriza por ser un secado de bajo costo, debido a que trabaja a presión atmosférica y temperaturas comparativamente bajas, y el hecho de que el enfriamiento simultáneo por evaporación y la transferencia de calor por convección permiten que se produzcan preparaciones bioactivas a temperaturas tan bajas como $30{ }^{\circ} \mathrm{C}$ (Bernaert et al., 2019).

Este método es utilizado para secar productos sensibles al calor como purés de frutas y vegetales, rebanadas, jugos en polvo, escamas u hojas. La retención del color, sabor, aroma, vitaminas y antioxidantes se pueden mejorar en este proceso de secado y la calidad del producto generalmente puede ser comparable o, en algunos casos, mejorar en comparación con liofilización. La técnica también se utiliza para secar productos farmacéuticos, nutracéuticos, cosméticos y pigmentos (Ortiz et al., 2015). 
En la tabla 4, se ha realizado la comparación de los métodos de secado (tratamientos térmicos) anteriormente expuestos, teniendo en cuenta diferentes parámetros, en los que se incluye la calidad del Aloe vera en función de la degradación de los compuestos funcionales.

\subsection{Discusión}

Según las tecnologías de secado estudiadas y teniendo en cuenta las características diferenciales en relación a tiempo, temperatura y costos de operación; es posible destacar y seleccionar el método más eficaz que permita preservar las propiedades funcionales del Aloe vera, teniendo en cuenta el gasto energético en el proceso de secado, el cual representa un $12-20 \%$ del total de las industrias manufactureras (Raghavi et al., 2018).

Los procesos de secado como: convección, aspersión, liofilización y ventana refractante se encuentran entre los más empleados; sin embargo, las condiciones aplicadas durante el proceso de secado pueden causar modificaciones irreversibles a los polisacáridos del Aloe vera, afectando su estructura original, lo que a su vez, puede conducir a cambios considerables, no solo en las propiedades fisiológicas y farmacológicas, sino también en el aspecto físico y propiedades químicas de los compuestos bioactivos de Aloe vera (R. Minjares et al., 2016). El secado por convección forzada es el método tradicional en el cual se puede regular la temperatura, pero el tiempo de operación es prolongado, dependiendo de la temperatura a trabajar y conociendo previamente la materia prima, para el caso Aloe vera, este material es sensible a altas temperaturas (termolábil). Por tanto, el incremento de un rango entre 50 a $90^{\circ} \mathrm{C}$ reduce el tiempo de secado desde 800 hasta 200 min llegando a la humedad de equilibrio, pero disminuye la capacidad antioxidante del gel (Domíguez et al., 2012); pero Miranda et al., (2009) sugiere que las temperaturas de secado para el gel de Aloe vera sea entre 60 y $70{ }^{\circ} \mathrm{C}$. No obstante, el secado con aire caliente es uno de los métodos de secado menos costosos en términos de consumo de energía y suministro de equipos, en comparación con otros procesos de deshidratación como secado por aspersión y liofilización (Ratti, 2013).
Por otra parte, el secado por aspersión, liofilización $\mathrm{y}$ ventana refractante se resaltan por preservar $\mathrm{y}$ obtener un producto de alta calidad, los cuales permiten conservar las propiedades nutracéuticas del Aloe vera para un posible uso industrial; sin embargo, según los parámetros mencionados anteriormente, el secado por ventana refractante presenta ventajas, como: evita la contaminación cruzada ya que el medio de transferencia de calor no entra en contacto directo con el producto, caracterizándose por tiempos de secado cortos (3-6 min) y temperaturas de secado relativamente bajas (entre $30-70^{\circ} \mathrm{C}$ ) y, por tanto, permite una mejor retención de color, vitaminas y antioxidantes (Bernaert et al., 2019). 
Tabla 4. Comparación de las técnicas de secado Fuente

\begin{tabular}{|c|c|c|c|c|}
\hline Parámetro & $\begin{array}{l}\text { Aire caliente } \\
\text { (convección) }\end{array}$ & Secado por aspersión & Liofilizado & Ventana refractante \\
\hline Tipo de producto & $\begin{array}{l}\text { Los más utilizados en la } \\
\text { industria de alimentos. Ají } \\
\text { dulce, uchuva }\end{array}$ & $\begin{array}{l}\text { Aloe vera, jugo de } \\
\text { naranja; pulpa de tomate, } \\
\text { cebada verde }\end{array}$ & $\begin{array}{l}\text { Aloe vera, fresa, frutos } \\
\text { tropicales }\end{array}$ & $\begin{array}{l}\text { Aloe vera, puré de zanahoria y } \\
\text { fresa, espárragos, frambuesa y } \\
\text { arándanos }\end{array}$ \\
\hline Operación & $\begin{array}{l}\text { Fácil de operar, sencillos, fácil } \\
\text { manejo }\end{array}$ & Fácil de operar & Complicado de operar & Fácil de operar \\
\hline Costo unidad & Unidades de bajo costo & Alta inversión inicial & $\begin{array}{l}\text { Unidad de alto costo, } \\
\text { costo de operación } \\
\text { cuatro veces más alto } \\
\text { que el secado tradicional }\end{array}$ & $\begin{array}{l}\text { Baratos en comparación con los } \\
\text { equipos de liofilización. } 1 / 3 \text { a } \\
1 / 2 \text { de un equipo liofilizador que } \\
\text { seca la misma cantidad de } \\
\text { producto }\end{array}$ \\
\hline $\begin{array}{l}\text { Pérdidas en el } \\
\text { proceso }\end{array}$ & No se presentan pérdidas & $\begin{array}{l}\text { Pérdida de la muestra, } \\
\text { adhesión a las paredes del } \\
\text { secador }\end{array}$ & $\begin{array}{l}\text { No se presentan } \\
\text { pérdidas }\end{array}$ & $\begin{array}{l}\text { Pérdidas por producto adherido } \\
\text { a la película }\end{array}$ \\
\hline $\begin{array}{l}\text { Nivel de } \\
\text { contaminación }\end{array}$ & $\begin{array}{l}\text { Depende de la fuente de } \\
\text { generación de aire. En } \\
\text { combustión directa se presenta }\end{array}$ & Contaminante & No contaminante & No contaminante \\
\hline
\end{tabular}


contaminación por los

productos de la combustión

\begin{tabular}{ccccc}
\hline $\begin{array}{c}\text { Costo de } \\
\text { mantenimiento }\end{array}$ & Bajo costo de mantenimiento & $\begin{array}{c}\text { Si se desea prevenir } \\
\text { pérdidas de producto, las } \\
\text { instalaciones de filtros de } \\
\text { sacos requieren alto costo. }\end{array}$ & --- & Bajo costo de mantenimiento \\
\hline $\begin{array}{c}\text { Calidad en productos } \\
\text { deshidratados de aloe } \\
\text { vera }\end{array}$ & $\begin{array}{c}\text { Modifica algunas propiedades } \\
\text { funcionales en el tejido de aloe } \\
\text { vera }\end{array}$ & $\begin{array}{c}\text { Deterioro del ingrediente } \\
\text { activo de aloe vera }\end{array}$ & $\begin{array}{c}\text { Preserva las propiedades } \\
\text { físicas y el ingrediente } \\
\text { activo en aloe vera }\end{array}$ & $\begin{array}{c}\text { Presenta buenas propiedades de } \\
\text { reconstitución, fluye libremente } \\
\text { y con atributos de color } \\
\text { aceptables. }\end{array}$ \\
\hline
\end{tabular}

(Mejia Terán, 2011) 
A partir de la liofilización, es posible obtener productos de alta calidad, debido a que la mayoría de reacciones que promueven el deterioro son lentas o en su defecto se detienen, pese a ello, el costo de producción es aproximadamente 8 y 4 veces mayor que el secado al aire convencional y el secado por aspersión, respectivamente. Por consiguiente, el alto costo operativo asociado con la liofilización restringe su uso solo a productos de alto valor (Ratti, 2013).

Finalmente, Minjares et al., (2017) y Medina et al., (2019), han manifestado que el uso de altas temperaturas (150 entrada $-75^{\circ} \mathrm{C}$ salida) para el secado por aspersión, es una de las principales ventajas, debido a que reduce la degradación de los compuestos bioactivos, derivado de los cortos tiempos de residencia $(<5 \mathrm{~s})$. Por lo tanto, este método ha sido ampliamente investigado y representa una excelente oportunidad por a su alto rendimiento de producción, bajo costo operativo (30-50 veces menos costoso que la liofilización) y el uso de equipos ampliamente disponibles para uso industrial en relación con las técnicas de deshidratación consultadas (Yingngam et $a l ., 2019)$.

\section{Conclusión}

Las técnicas de secado que mejor se ajustan a las necesidades de conservar las propiedades funcionales del Aloe vera, es la liofilización y ventanas refractantes, dado que permiten emplear bajas temperaturas $\left(30-70^{\circ} \mathrm{C}\right)$ y tiempos cortos de secado $(3$ - 6 min). También, evita la contaminación cruzada, preserva las propiedades físicas (color), componentes activos: vitaminas y antioxidantes. Los principales factores que se deben tener en cuenta para la conservación de las propiedades funcionales del aloe son temperatura y tiempos de exposición, ya que, a mayores, ésta es directamente proporcional a su degradación.

\section{Referencias bibliográficas}

Ahlawat, K. S., \& Khatkar, B. S. (2011). Processing, food applications and safety of aloe vera products: A review. Journal of Food Science and Technology, 48(5), 525-533. https://doi.org/10.1007/s13197-011-0229-z
Amanullah, S., Jahangir, M. M., Ikram, R. M., Sajid, M., Abbas, F., \& Mallano, A. I. (2017). Aloe vera Coating Efficiency on Shelf Life of Eggplants at Differential Storage Temperatures. Journal of Northeast Agricultural University (English Edition), 23(4), 15-25. https://doi.org/10.1016/s1006-8104(17)30003-x

Bahmani, M., Shahinfard, N., Fasihzadeh, S., Mirhosseini, M., \& Rafieian-Kopaei. (2016). Aloe vera: An update on its phytomedicinal, pharmaceutical and therapeutic properties. Der Pharmacia Lettre, 8(1), 206-213.

Bajpai, S. (2018). Biological importance of Aloe vera and its active constituents. In Synthesis of Medicinal Agents from Plants (Vol. 1753). Elsevier Ltd. https://doi.org/10.1016/b978-0-08102071-5.00008-8

Baruah, A., Bordoloi, M., \& Deka Baruah, H. P. (2016). Aloe vera: A multipurpose industrial crop. Industrial Crops and Products, 94, 951963. https://doi.org/10.1016/j.indcrop.2016.08.034

Benítez, S., Achaerandio, I., Pujolà, M., \& Sepulcre, F. (2015). Aloe vera as an alternative to traditional edible coatings used in fresh-cut fruits: A case of study with kiwifruit slices. LWT - Food Science and Technology, 61(1), 184-193.

https://doi.org/10.1016/j.lwt.2014.11.036

Berk, Z. (2018). Freeze drying (lyophilization) and freeze concentration. In Food Process Engineering and Technology (3rd ed., pp. 567581). Academic Press. https://doi.org/10.1016/b978-0-12-8120187.00023-3

Bernaert, N., Van Droogenbroeck, B., Van Pamel, E., \& De Ruyck, H. (2019). Innovative refractance window drying technology to keep nutrient value during processing. Trends in Food Science and Technology, 84(June 2017), 22-24. https://doi.org/10.1016/j.tifs.2018.07.029

Bialik-Wąs, K., Pluta, K., Malina, D., Barczewski, M., Malarz, K., \& Mrozek-Wilczkiewicz, A. (2021). Advanced SA/PVA-based hydrogel matrices with prolonged release of Aloe vera as promising wound dressings. Materials Science 
and Engineering C, 120(August 2020). https://doi.org/10.1016/j.msec.2020.111667

Canche-Escamilla, G., Colli-Acevedo, P., BorgesArgaez, R., Quintana-Owen, P., May-Crespo, J. F., Cáceres-Farfan, M., Yam Puc, J. A., Sansores-Peraza, P., \& Vera-Ku, B. M. (2019). Extraction of phenolic components from an Aloe vera (Aloe barbadensis Miller) crop and their potential as antimicrobials and textile dyes. Sustainable Chemistry and Pharmacy, 14(August). https://doi.org/10.1016/j.scp.2019.100168

Castro, A. M., Mayorga, E. Y., \& Moreno, F. L. (2018). Mathematical modelling of convective drying of fruits: A review. Journal of Food Engineering, 223, 152-167. https://doi.org/10.1016/j.jfoodeng.2017.12.012

Cervantes, C. V., Medina-Torres, L., GonzálezLaredo, R. F., Calderas, F., Sánchez-Olivares, G., Herrera-Valencia, E. E., Gallegos Infante, J. A., Rocha-Guzman, N. E., \& RodríguezRamírez, J. (2014). Study of spray drying of the Aloe vera mucilage (Aloe vera barbadensis Miller) as a function of its rheological properties. LWT - Food Science and Technology, 55(2), 426-435. https://doi.org/10.1016/j.lwt.2013.09.026

Dehnad, D., Jafari, S. M., \& Afrasiabi, M. (2016). Influence of drying on functional properties of food biopolymers: From traditional to novel dehydration techniques. Trends in Food Science and Technology, 57, 116-131. https://doi.org/10.1016/j.tifs.2016.09.002

Domíguez, R., Arzate, I., Chanona, J., Welti, J., Alvarado, J., Garibay, V., \& Gutiérrez, G. (2012). El gel de aloe vera: Estructura, composición quimica,procesameinto, actividad biológica e importancia en la industria farmacéutica y alimentaria. Revista Mexicana de Ingeniera Qumica, 11(1), 23-43.

Franco, S., Jaques, A., Pinto, M., Fardella, M., Valencia, P., Núñez, H., Ramírez, C., \& Simpson, R. (2019). Dehydration of salmon (Atlantic salmon), beef, and apple (Granny Smith) using Refractance window ${ }^{\mathrm{TM}}$ : Effect on diffusion behavior, texture, and color changes. Innovative Food Science and Emerging
Technologies, 52(November 2018), 8-16. https://doi.org/10.1016/j.ifset.2018.12.001

Guillén, F., Díaz-Mula, H. M., Zapata, P. J., Valero, D., Serrano, M., Castillo, S., \& MartínezRomero, D. (2013). Aloe arborescens and Aloe vera gels as coatings in delaying postharvest ripening in peach and plum fruit. Postharvest Biology and Technology, 83, 54-57. https://doi.org/10.1016/j.postharvbio.2013.03.0 11

Horn, J., Schanda, J., \& Friess, W. (2018). Impact of fast and conservative freeze-drying on product quality of protein-mannitol-sucrose-glycerol lyophilizates. European Journal of Pharmaceutics and Biopharmaceutics, 127(March), 342-354. https://doi.org/10.1016/j.ejpb.2018.03.003

Javed, S., \& Atta, R. (2014). Aloe vera gel in food, health products, and cosmetics industry. In Studies in Natural Products Chemistry (1st ed., Vol. 41). Elsevier B.V. https://doi.org/10.1016/B978-0-444-632944.00009-7

Karam, M. C., Petit, J., Zimmer, D., Baudelaire Djantou, E., \& Scher, J. (2016). Effects of drying and grinding in production of fruit and vegetable powders: A review. Journal of Food Engineering, 188, 32-49.

https://doi.org/10.1016/j.jfoodeng.2016.05.001

Khaliq, G., Ramzan, M., \& Baloch, A. H. (2019). Effect of Aloe vera gel coating enriched with Fagonia indica plant extract on physicochemical and antioxidant activity of sapodilla fruit during postharvest storage. Food Chemistry, 286(October 2018), 346-353. https://doi.org/10.1016/j.foodchem.2019.01.135

Khorasani, S., Danaei, M., \& Mozafari, M. R. (2018). Nanoliposome technology for the food and nutraceutical industries. Trends in Food Science and Technology, 79(February), 106-115. https://doi.org/10.1016/j.tifs.2018.07.009

Krokida, M., Pappa, A., \& Agalioti, M. (2012). Effect of drying on Aloe's functional components. Procedia Food Science, 1, 15231527. https://doi.org/10.1016/j.profoo.2011.09.225 
Lacerda, G. E. (2016). Composição química, fitoquímica e dosagem de metais pesados das cascas das folhas secas e do gel liofilizado de Aloe Vera cultivadas em hortas comunitárias da cidade de palmas, Tocantins.

Lozano, L. A., Muvdi, C., \& Mejia, luz D. (2011). Estabilización del gel de Aloe Barbadensis Miller y disminución de su concentración por adsorción en columna con carbón activado. 24(1), 61-67.

Maan, A. A., Nazir, A., Khan, M. K. I., Ahmad, T., Zia, R., Murid, M., \& Abrar, M. (2018). The therapeutic properties and applications of Aloe vera: A review. Journal of Herbal Medicine, 12(January), 1-10.

https://doi.org/10.1016/j.hermed.2018.01.002

Medina, L., Calderas, F., Minjares, R., Femenia, A., Sánchez-Olivares, G., Gónzalez-Laredo, F. R., Santiago-Adame, R., Ramirez-Nuñez, D. M., Rodríguez-Ramírez, J., \& Manero, O. (2016). Structure preservation of Aloe vera (barbadensis Miller) mucilage in a spray drying process. $L W T$ - Food Science and Technology, 66, 93-100. https://doi.org/10.1016/j.lwt.2015.10.023

Medina, L., Núñez, D. M., Calderas, F., \& González, R. F. (2019). Industrial Crops \& Products Microencapsulation of gallic acid by spray drying with aloe vera mucilage ( aloe barbadensis miller ) as wall material. Industrial Crops \& Products, 138(June), 111461. https://doi.org/10.1016/j.indcrop.2019.06.024

Mejia Terán, A. L. (2011). Efecto de la deshidratación por radiación infrarroja sobre algunas características fisicoquímicas de interés comercial del Aloe vera (Aloe barbadensis). 77.

http://intellectum.unisabana.edu.co/handle/1081 $8 / 1249$ ?locale-attribute $=$ en

Minjares, R., \& Femenia, A. (2019). Aloe vera. In Journal of the American Academy of Dermatology (Vol. 18, Issue 4, pp. 714-720). Elsevier Inc. https://doi.org/10.1016/S01909622(88)70095-X

Minjares, R., Femenia, A., Comas-Serra, F., Rosselló, C., Rodríguez-González, V. M., GonzálezLaredo, R. F., Gallegos-Infante, J. A., \&
Medina-Torres, L. (2016). Effect of different drying procedures on physicochemical properties and flow behavior of Aloe vera (Aloe barbadensis Miller) gel. LWT - Food Science and Technology, 74, 378-386. https://doi.org/10.1016/j.lwt.2016.07.060

Minjares, Rafael, Rodríguez, V. M., González, R. F., Eim, V., González, M. R., \& Femenia, A. (2017). Effect of different drying procedures on the bioactive polysaccharide acemannan from Aloe vera (Aloe barbadensis Miller ). Carbohydrate Polymers, 168, 327-336. https://doi.org/10.1016/j.carbpol.2017.03.087

Miranda, M., Maureira, H., Rodríguez, K., \& Vegagálvez, A. (2009). Influence of temperature on the drying kinetics, physicochemical properties , and antioxidant capacity of Aloe Vera (Aloe Barbadensis Miller ) gel. Journal of Food Engineering, 91(2), 297-304. https://doi.org/10.1016/j.jfoodeng.2008.09.007

Mishra, L. K., \& Sangma, D. (2017). Quality attributes, phytochemical profile and storage stability studies of functional ready to serve (RTS) drink made from blend of Aloe vera, sweet lime, amla and ginger. Journal of Food Science and Technology, 54(3), 761-769. https://doi.org/10.1007/s13197-017-2516-9

Mudgil, D., Barak, S., \& Darji, P. (2016).

Development and characterization of functional cultured buttermilk utilizing Aloe vera juice. Food Bioscience, 15, 105-109. https://doi.org/10.1016/j.fbio.2016.06.001

Nazir, A., Khan, K., Maan, A., Zia, R., Giorno, L., \& Schroën, K. (2019). Membrane separation technology for the recovery of nutraceuticals from food industrial streams. Trends in Food Science and Technology, 86(June 2018), 426438. https://doi.org/10.1016/j.tifs.2019.02.049

Nejatzadeh, F. (2013). Antibacterial activities and antioxidant capacity of Aloe vera. Organic and Medicinal Chemistry Letters, 3(1), 5. https://doi.org/10.1186/2191-2858-3-5

Nicolau-Lapeña, I., Colàs-Medà, P., Alegre, I., Aguiló-Aguayo, I., Muranyi, P., \& Viñas, I. (2021). Aloe vera gel: An update on its use as a functional edible coating to preserve fruits and 
vegetables. Progress in Organic Coatings, 151(November 2020).

https://doi.org/10.1016/j.porgcoat.2020.106007

Nindo, C. I., Powers, J. R., \& Tang, J. (2010). Thermal properties of Aloe vera powder and rheology of reconstituted gels. Transactions of the ASABE, 53(4), 1193-1200.

Ortega, R., Collazo Bigliardi, S., Roselló, J., Santamarina, P., \& Chiralt, A. (2017). Antifungal starch-based edible films containing Aloe vera. Food Hydrocolloids, 72, 1-10. https://doi.org/10.1016/j.foodhyd.2017.05.023

Ortiz, M. J., Gulati, T., Datta, A. K., \& OchoaMartínez, C. I. (2015). Quantitative understanding of Refractance Window ${ }^{\mathrm{TM}}$ drying. Food and Bioproducts Processing, 95, 237-253.

https://doi.org/10.1016/j.fbp.2015.05.010

Patiño, M. J. (2016). Identificación del nivel de industrialización del Aloe vera en Colombia. Universidad de la Salle.

Raghavi, L. M., Moses, J. A., \& Anandharamakrishnan, C. (2018). Refractance window drying of foods: A review. Journal of Food Engineering, 222, 267-275.

https://doi.org/10.1016/j.jfoodeng.2017.11.032

Rahman, S., Carter, P., \& Bhattarai, N. (2017). Aloe Vera for Tissue Engineering Applications. https://doi.org/10.3390/jfb8010006

Ramachandra, C. T., \& Srinivasa, P. (2008). Processing of Aloe vera leaf gel: A review. American Journal of Agricultural and Biological Science, 3(2), 502-510.

Ratti, C. (2013). Freeze drying for food powder production. In Handbook of food powders : Processes and properties. Woodhead Publishing Limited. https://doi.org/10.1533/9780857098672.1.57

Ray, A. (2012). Scope of Aloe vera as Medicinal Plant and Skin Care. 3(June), 1-3.

Reyes, J. E., Guanoquiza, M. I., Tabilo-Munizaga, G., Vega-Galvez, A., Miranda, M., \& PérezWon, M. (2012). Microbiological stabilization of Aloe vera (Aloe barbadensis Miller) gel by high hydrostatic pressure treatment.

International Journal of Food Microbiology, 158(3), 218-224. https://doi.org/10.1016/j.ijfoodmicro.2012.07.0 19

Rosero, I. M. (2018). Comparación de técnicas de secado ventana Refractiva y Convección forzada en frutas tropicales. UNIVERSIDAD PONTIFICIA BOLIVARIANA.

Sabarez, H. (2016). Drying of Food Materials. In Reference Module in Food Science. Elsevier. https://doi.org/10.1016/B978-0-08-1005965.03416-8

Sahu, P. K., Dayal Giri, D., Singh, R., Pandey, P., Gupta, S., Kumar Shrivastava, A., Kumar, A., \& Dev Pandey, K. (2013). Therapeutic and Medicinal Uses of Aloe vera: A Review. Pharmacology \& Pharmacy, 4, 599-610. https://doi.org/10.4236/pp.2013.48086

Sánchez, D. I., López, J., Sendón, R., \& Sanches, A. (2017). Aloe vera: Ancient knowledge with new frontiers. Trends in Food Science and Technology, 61, 94-102. https://doi.org/10.1016/j.tifs.2016.12.005

Santacruz, V., Santacruz, C., \& Laguna, J. O. (2015). Physical characterization of freeze-dried foam prepared from aloe vera gel and guar gum. Revista Vitae, 22(2), 75-86. https://doi.org/10.17533/udea.vitae.v22n2a02

Shishir, M. R. I., \& Chen, W. (2017). Trends of spray drying: A critical review on drying of fruit and vegetable juices. Trends in Food Science and Technology, 65, 49-67. https://doi.org/10.1016/j.tifs.2017.05.006

Solek, P., Majchrowicz, L., \& Koziorowski, M. (2018). Aloe arborescens juice prevents EMFinduced oxidative stress and thus protects from pathophysiology in the male reproductive system in vitro. Environmental Research, 166(May), 141-149. https://doi.org/10.1016/j.envres.2018.05.035

Sriariyakul, W., Swasdisevi, T., Devahastin, S., \& Soponronnarit, S. (2016). Drying of aloe vera puree using hot air in combination with farinfrared radiation and high-voltage electric 
field: Drying kinetics, energy consumption and product quality evaluation. Food and Bioproducts Processing, 100, 391-400. https://doi.org/10.1016/j.fbp.2016.08.012

Srujana, P., Kishore, V. S., Srikanth, K., \& Shanmukha, P. Y. (2012). Aloe vera - A Review. 4(2), 119-123.

Szadzińska, J., Kowalski, S. J., \& Stasiak, M. (2016). Microwave and ultrasound enhancement of convective drying of strawberries: Experimental and modeling efficiency. International Journal of Heat and Mass Transfer, 103, 1065-1074. https://doi.org/10.1016/j.ijheatmasstransfer.201 6.08 .001

Vega, A., Uribe, E., Lemus, R., \& Miranda, M. (2007). Hot-air drying characteristics of Aloe vera (Aloe barbadensis Miller) and influence of temperature on kinetic parameters. $L W T$ - Food Science and Technology, 40(10), 1698-1707. https://doi.org/10.1016/j.lwt.2007.01.001

Vieira, J. M., Flores-López, M. L., de Rodríguez, D. J., Sousa, M. C., Vicente, A. A., \& Martins, J. T. (2016). Effect of chitosan-Aloe vera coating on postharvest quality of blueberry (Vaccinium corymbosum) fruit. Postharvest Biology and Technology, 116, 88-97. https://doi.org/10.1016/j.postharvbio.2016.01.0 11

Yingngam, B., Kacha, W., Rungseevijitprapa, W., \& Sudta, P. (2019). Response surface optimization of spray-dried citronella oil microcapsules with reduced volatility and irritation for cosmetic textile uses. Powder Technology, 355, 372-385. https://doi.org/10.1016/j.powtec.2019.07.065 